\title{
Waveguide Arrays as Plasmonic Metamaterials: Transmission below Cutoff
}

\author{
Alastair P. Hibbins, Matthew J. Lockyear, Ian R. Hooper, and J. Roy Sambles \\ School of Physics, University of Exeter, Stocker Road, Exeter EX4 4QL, United Kingdom
}

(Received 21 September 2005; published 23 February 2006)

\begin{abstract}
Since the work of Ebbesen et al. [Nature (London) 391, 667 (1998)], there has been immense interest in the optical properties of subwavelength holes in metal layers. While the enhanced transmission observed is generally associated with surface plasmon polaritons (SPPs), theoretical predictions suggest a similar response with perfectly conducting materials. However, Pendry et al. [Science 305, 847 (2004)] proposed that, if textured on a subwavelength scale, even perfect conductors support surface modes. Here, using microwave radiation incident upon an array of metal waveguides, we observe peaks in the transmissivity below cutoff and confirm the crucial role of these SPP-like modes in the mechanism responsible.
\end{abstract}

DOI: 10.1103/PhysRevLett.96.073904

At the interface between a metal and a dielectric, there exists a bound electromagnetic (EM) surface wave. In the visible regime, this mode is known as a surface plasmon polariton (SPP) characterized by strongly enhanced fields at the interface that decay exponentially with distance into the bounding media on either side $\left(\sim 10^{-2} \lambda\right.$ in the metal and $\sim 10^{-1} \lambda$ in the dielectric) [1-3]. The SPP has in-plane momentum greater than that of the light in the adjacent dielectric, and so, in order to resonantly excite it, the inplane momentum of the incident radiation must be suitably increased. One way of doing this is through a periodic surface, a diffraction grating. The regular lattice provides the necessary increase of the in-plane wave vector via scattering of the incident EM wave by integer multiples of the grating wave vector $2 \pi / d$, where $d$ is the lattice spacing (grating pitch) [2].

At lower frequencies, metals more effectively screen the incident fields. Indeed, in the microwave regime, the penetration of the fields into a planar substrate is only $\sim 10^{-4} \lambda$, yet they extend many hundreds of wavelengths above. This surface mode can, therefore, no longer be regarded as strongly bound to the interface and is more often described as a surface current. Nevertheless, Pendry, Martín-Moreno, and García-Vidal $[4,5]$ have recently shown that even in the perfectly conducting limit, surfaces can support modes that act like strongly localized SPPs if the surfaces are textured on a subwavelength scale. Such a structure needs to be considered as a metamaterial [6], where the indentations (e.g., holes, dimples, grooves, etc.) allow some of the field to penetrate into the substrate, hence modifying the field matching conditions at its surface. As an example, consider an assembly of deep holes. The voids will act as waveguides with a cutoff frequency $\nu_{\text {cutoff }}$, below which no propagating modes are allowed. Hence, below this limit only evanescent fields exist within the substrate. Crucially, it is exactly this field characteristic that must be met for a surface mode to be supported. Furthermore, if the holes are arranged periodically, then the in-plane momentum of the incident radiation may be increased, thereby permitting coupling between the incident radiation and the mode
PACS numbers: 42.25.Bs, 42.79.Dj, 73.20.Mf, 84.40.Az

when the necessary momentum matching condition is met $[2,7]$.

Until relatively recently, it was generally believed impossible to transmit a significant intensity of EM radiation through features or gaps smaller than the wavelength illuminating them. However, the seminal results that Ebbesen et al. first published in 1998 [8] proved that it was possible to break this diffraction limit. A gold foil perforated with an array of holes, each $150 \mathrm{~nm}$ across, not only transmitted visible radiation but actually transmitted more radiation than was directly incident on the voids. These remarkable results were initially attributed to plasmonics [9]. However, the physical understanding of the mechanism responsible was put in doubt when subsequent numerical predictions [10] confirmed that the phenomenon should still be observed when perfect conductors were used-even though no bound surface wave was thought to exist. The aforementioned proposal of Pendry and co-workers [4,5] was that a structured perfectly conducting surface may support SPP-like waves that behave in a similar manner to those known to exist on real metals. In the present study, we use microwave measurements and finite element method (FEM) modeling [11] not only to verify the existence of this new class of mode but also to confirm its role in enhanced transmission observed for perforated, perfectly conducting layers.

Our sample (Fig. 1) is formed from a $350 \times 350 \mathrm{~mm}$ array of hollow, square-ended brass tubes. The inner size of the tubes $(a=6.96 \mathrm{~mm})$ is chosen so that the cutoff frequency lies in the microwave regime, $\nu_{\text {cutoff }}=c / 2 a=$ $21.54 \mathrm{GHz}$. Note that $\nu_{\text {cutoff }}$ is for waveguides of infinite length and as such ignores the additional requirement for momentum conservation in the $z$ direction for finite length guides. We use tubes of length $L=15.0 \mathrm{~mm}$, and, as we shall subsequently show, the lowest frequency waveguide resonance is centered on $22.8 \mathrm{GHz}$, representing the true cutoff frequency $\left(\nu_{\text {cutoff }}^{\prime}\right)$. It is also important to note that most hole-array transmission studies have been undertaken with $d>2 a$. Therefore, if SPP-like modes associated with the structure of the substrate are indeed playing a role in the 


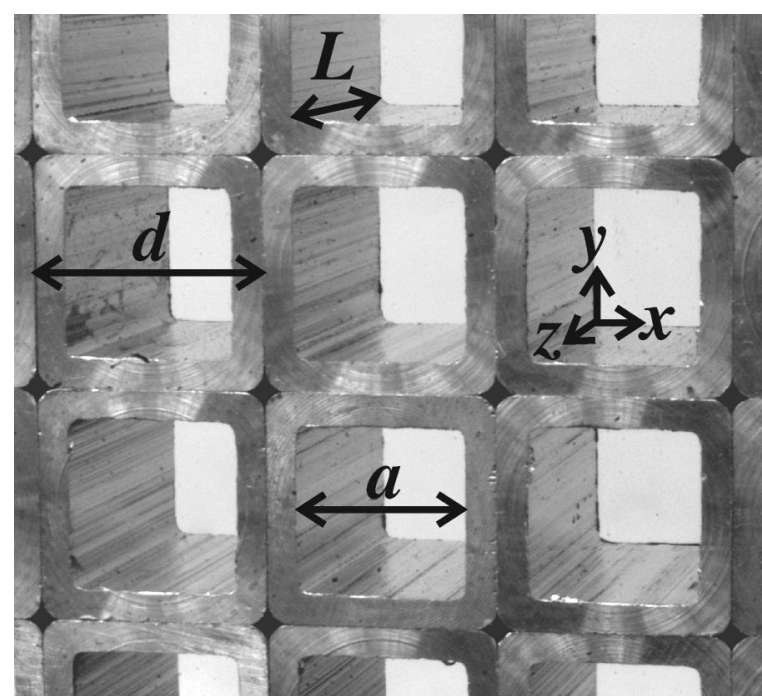

FIG. 1. Photograph of part of the sample used in the present study $(d=9.53 \mathrm{~mm}, a=6.96 \mathrm{~mm})$. The coordinate system is also shown.

transmission mechanism, then we would expect to see only a rather featureless dispersion curve in this regime. This is because the frequency to which the surface mode should be asymptotic ( $\left.\sim \nu_{\text {cutoff }}^{\prime} \geq c / 2 a[4]\right)$ is greater than that corresponding to the upper limit of the onset of diffraction (at normal incidence, $\nu_{\text {diffraction }}=c / d$ ), and, hence, no significant deviation from the diffracted light line is observed. In other words, we would expect to record a resonant transmission peak at a frequency just below the diffraction edge (e.g., [12-14]). However, in the present study we choose $d=9.53 \mathrm{~mm}<2 a$, and, hence, the cutoff frequency is lower than the onset of diffraction at normal incidence. Therefore, by recording the transmission spectra as a function of the angle of incidence, we are able to confirm the role of this surface mode in the transmission by mapping the mode's dispersion and witnessing the characteristic asymptotic limit.

Our experimental results are shown in Fig. 2. By positioning the transmitting horn antenna at the focus of a 4meter radius-of-curvature mirror, we provide a collimated beam. Microwave radiation $(18.0 \mathrm{GHz} \leq \nu \leq 26.5 \mathrm{GHz})$ is incident upon the sample in the $x z$ plane, and the nondiffracted transmitted beam is subsequently collected by a similar mirror and focused onto a detector via a second horn antenna. The orientations of both horn antennas are set to pass only transverse-magnetic (TM- or $p$-) polarized radiation. By placing the sample on a computer-controlled table, we are able to rotate it around the $z$ axis and, hence, record the transmission data as a function of frequency $\nu$ and angle $\theta$. The solid gray lines on the gray scale plot represent the positions of peak transmission and, hence, give an indication of the band structure of the sample. The dotted-dashed lines correspond to angles of incidence $(\theta=$ $5^{\circ}, 21^{\circ}$, and $26^{\circ}$ ) for which spectra are plotted in Fig. 2(b). The predictions from the FEM numerical model at these
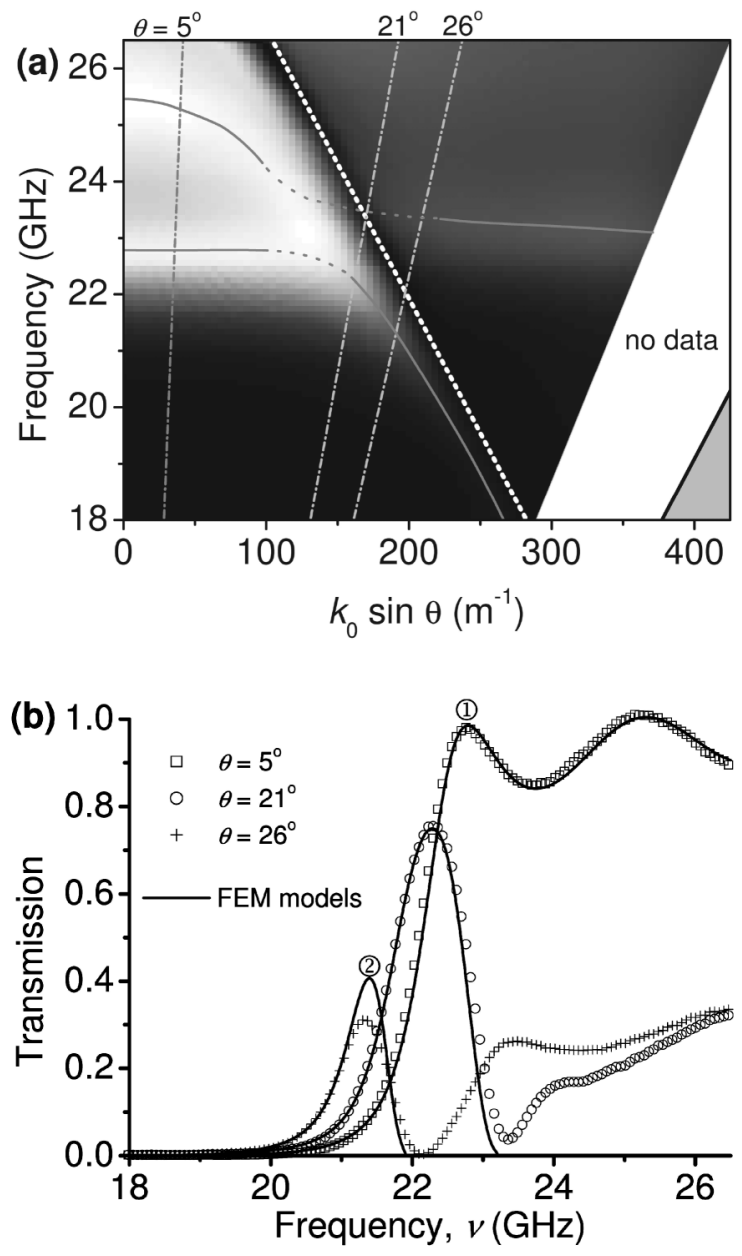

FIG. 2. (a) Intensity of the nondiffracted transmitted beam (gray scale plotted on a linear scale: black $T=0.0$, white $T=$ 1.0) as a function of frequency $\nu$ and in-plane wave vector $k_{x}=$ $2 \pi \nu \sin \theta / c$. The shaded region in the bottom right-hand corner corresponds to momentum space within which modes may not be directly coupled to as they are beyond the light line (solid black line). The white dotted line represents the first-order diffracted light line associated with the periodicity of the array. Data are recorded only for angles of incidence up to $50^{\circ}$. As a guide to the eye, the solid gray lines provide a trace of the position of the transmission peaks associated with each mode. The dotted segments provide an estimation of the band structure in the region where the transmission peaks are not a good indication of the mode position. (b) Nondiffracted transmission spectra recorded from the sample at angles of $\theta=5^{\circ}, 21^{\circ}$, and $26^{\circ}$ [illustrated by gray dotted-dashed lines in (a)], together with the predictions from the numerical model. In order to reduce the complexity of the problem, model solutions are requested only at frequencies below the onset of diffraction (at 29.0, 23.2, and $21.9 \mathrm{GHz}$ for $\theta=5^{\circ}, 21^{\circ}$, and $26^{\circ}$, respectively). The electric field distributions associated with the resonances labeled (1) and (2) are shown in Fig. 3.

angles are also presented and show excellent agreement with the experimental data.

Let us first consider the $\theta=5^{\circ}$ spectrum. The electric field distribution in the $x z$ plane associated with the trans- 

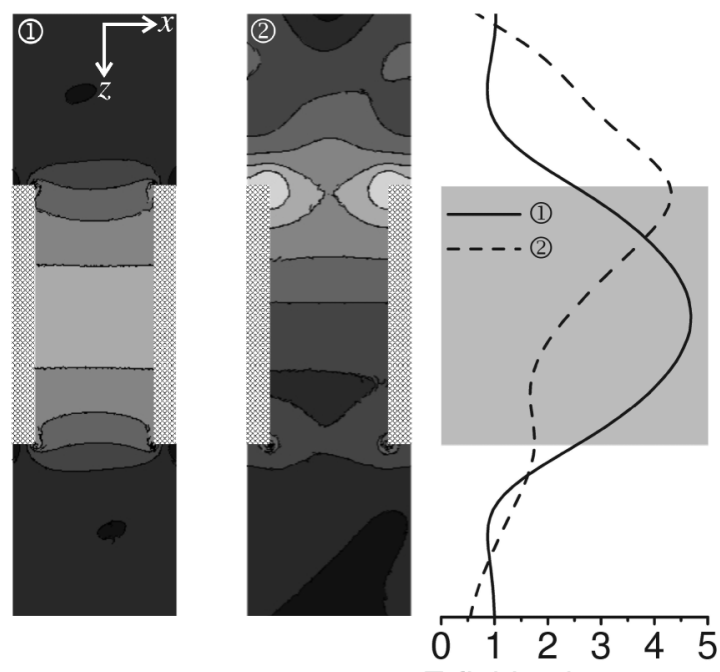

E-field enhancement

FIG. 3. Predictions of the time-averaged electric field enhancements of the resonances labeled (1) $\left(\theta=5^{\circ}, 22.8 \mathrm{GHz}\right)$ and (2) $\left(\theta=26^{\circ}, 21.4 \mathrm{GHz}\right)$. In the gray scale plots, the lightest shading corresponds to field enhancements of six times and greater. TMpolarized radiation is incident in the $x z$ plane from above. The line graph illustrates the same quantities plotted along a line in the $z$ direction through the center of a tube. The shaded area corresponds to the region inside the cavity.

mission peak at $22.8 \mathrm{GHz}$ (labeled (1) is plotted in Fig. 3. By also considering its field solution in the $x y$ plane (not shown) and counting the number of field maxima along the tube length $(N=1)$, we can identify this mode as the lowest frequency standing wave resonance of the $\mathrm{TE}_{10}$ waveguide mode [15]. This frequency therefore presents a limit below which only evanescent fields can exist within the tubes. Clearly, a whole family of transmission resonances associated with standing wave solutions are observed above this cutoff; for example, the transmission peak at $25.3 \mathrm{GHz}$ corresponds to the $N=2$ resonance of the same mode (fields not shown). These resonances are all the result of diffractive coupling via the periodicity of the array and, therefore, demonstrate strong dispersive character close to the onset of diffraction. Indeed, the band structure is analogous in many ways to the flat-banded TEM waveguide modes observed on deep and narrow grating grooves [16]; however, unlike the TE modes supported by the tubes in the present study, TEM modes have no lower frequency cutoff. Nevertheless, they demonstrate similar dispersion characteristics, anticrossing with the light lines. As the angle of incidence is increased, the frequency of the $N=2$ resonance begins to fall almost immediately, while the $N=1$ mode is relatively unperturbed up to $\theta \approx 15^{\circ}$. The two bands then begin to interact and become indistinguishable. In this region, the band structure can no longer be accurately determined from positions of transmission peaks, because not only do their characters become mixed, but also the shape of the resonant peaks becomes distorted by the dominance of the diffraction feature. We indicate the expected band structure in this region with a broken gray line [16]. It is clear that the higher frequency mode morphs into an $N=1$ resonance as it moves into the diffracting region (fields not shown), while the lower mode follows the diffracted light line as it decreases in frequency. Since the tubes no longer support propagating modes, the transmission intensity associated with the lower frequency resonance begins to weaken, and its fields become evanescent inside the cavity. Consider the transmission peak at $\sim 21.4 \mathrm{GHz}$ in the $\theta=$ $26^{\circ}$ spectrum (2). The electric field predictions confirm that it is not associated with a waveguide mode (Fig. 3). Instead, it shows strongly enhanced electric fields at the illuminated surface of the array that exponentially decay along the length of the cavity before strengthening again close to the bottom face of the structure. We associate this field distribution with the excitation of a diffractively coupled SPP-like mode on the upper surface whose fields decay along the length of the waveguide. The fields are, nevertheless, intense enough to couple to an identical mode on the lower face that, in turn, diffractively couples out to free radiation.

In conclusion, we have studied the microwave transmission of an array of square-ended near-perfectly conducting waveguides. Our experimental data show strong resonant transmission of EM radiation below the cutoff frequency of the waveguides, and the spectra agree well with the predictions from a FEM model. By working in the regime around this cutoff frequency, we have been able to map out the dispersion of the mode. Modeling of the field solutions on resonance has shown the mode responsible for the subcutoff transmission to be the recently proposed SPP-like mode, and its dispersion asymptotically approaches the frequency predicted [4-6]. In addition, our study resolves a long-standing debate, proving beyond doubt that surface modes are responsible for the "anomalous" transmission phenomenon though arrays of holes in metal layers even in the limit of perfect conductivity.

The authors acknowledge the financial support of the Engineering and Physical Sciences Research Council (EPSRC, United Kingdom). We also thank Pete Cann for his assistance in making the sample.

[1] R. H. Ritchie, Phys. Rev. 106, 874 (1957).

[2] H. Raether, Surface Plasmons (Springer-Verlag, Berlin, 1988), Chap. 2.

[3] W. L. Barnes, A. Dereux, and T.W. Ebbesen, Nature (London) 424, 824 (2003).

[4] J. B. Pendry, L. Martín-Moreno, and F. J. García-Vidal, Science 305, 847 (2004).

[5] F. J. Garcia-Vidal, L. Martín-Moreno, and J. B. Pendry, J. Opt. A Pure Appl. Opt. 7, S97 (2005).

[6] D. R. Smith, J. B. Pendry, and M. C. K. Wiltshire, Science 305, 788 (2004).

[7] A. P. Hibbins, B. R. Evans, and J. R. Sambles, Science 308, 670 (2005). 
[8] T. W. Ebbesen, H. J. Lezec, H. Ghaemi, T. Thio, and P. A. Wolff, Nature (London) 391, 667 (1998).

[9] R. Sambles, Nature (London) 391, 641 (1998).

[10] L. Martín-Moreno, F. J. García-Vidal, H. J. Lezec, K. M. Pellerin, T. Thio, J. B. Pendry, and T. W. Ebbesen, Phys. Rev. Lett. 86, 1114 (2001).

[11] HFSS, Ansoft Corporation, Pittsburgh, PA, USA.

[12] H.F. Ghaemi, T. Thio, D. E. Grupp, T. W. Ebbesen, and H. J. Lezec, Phys. Rev. B 58, 6779 (1998).
[13] J. Gómez Rivas, C. Schotsch, P. Haring Bolivar, and H. Kurz, Phys. Rev. B 68, 201306(R) (2003).

[14] M. Beruete, M. Sorolla, I. Campillo, J. S. Dolado, L. Martín-Moreno, and F. J. García-Vidal, Opt. Lett. 29, 2500 (2004).

[15] D. M. Pozar, Microwave Engineering (Wiley, New York, 1998).

[16] I. R. Hooper and J. R. Sambles, Phys. Rev. B 66, 205408 (2002). 